University of Nebraska - Lincoln

DigitalCommons@University of Nebraska - Lincoln

\title{
Dipole-Induced, Thermally Stable Lamellar Structure by Polar Aromatic Silane
}

\author{
Jinyue Jiang \\ University of Nebraska-Lincoln, jjiang2@unl.edu \\ Ocelio V. Lima \\ University of Nebraska-Lincoln \\ Yong Pei \\ University of Nebraska-Lincoln \\ Xiao Cheng Zeng \\ University of Nebraska-Lincoln, xzeng1@unl.edu \\ Li Tan \\ University of Nebraska-Lincoln, Itan4@unl.edu \\ See next page for additional authors
}

Follow this and additional works at: https://digitalcommons.unl.edu/chemzeng

Part of the Chemistry Commons

Jiang, Jinyue; Lima, Ocelio V.; Pei, Yong; Zeng, Xiao Cheng; Tan, Li; and Forsythe, Eric, "Dipole-Induced, Thermally Stable Lamellar Structure by Polar Aromatic Silane" (2009). Xiao Cheng Zeng Publications. 96. https://digitalcommons.unl.edu/chemzeng/96

This Article is brought to you for free and open access by the Published Research - Department of Chemistry at DigitalCommons@University of Nebraska - Lincoln. It has been accepted for inclusion in Xiao Cheng Zeng Publications by an authorized administrator of DigitalCommons@University of Nebraska - Lincoln. 


\section{Authors}

Jinyue Jiang, Ocelio V. Lima, Yong Pei, Xiao Cheng Zeng, Li Tan, and Eric Forsythe 


\title{
Dipole-Induced, Thermally Stable Lamellar Structure by Polar Aromatic Silane
}

\author{
Jinyue Jiang, ${ }^{\dagger}$ Ocelio V. Lima, ${ }^{\dagger}$ Yong Pei, ${ }^{\ddagger}$ Xiao Cheng Zeng, ${ }^{*, \ddagger} \mathrm{Li}$ Tan, ${ }^{*, \dagger}$ and Eric Forsythe ${ }^{*, \S}$ \\ Department of Engineering Mechanics and Nebraska Center for Materials and Nanoscience, University of \\ Nebraska, Lincoln, Nebraska 68588, Department of Chemistry, University of Nebraska, Lincoln, Nebraska 68588, \\ and Flexbile Display Center, Army Research Laboratory, Adelphi, Maryland, 20783-1197
}

Received October 14, 2008; E-mail: Itan4@unl.edu; xzeng1@unl.edu; eric.forsythe@us.army.mil

Lamellar structures, consisting of alternating inorganic layers separated by organic moieties, have attracted much attention over a wide range of applications, such as polymer-clay composites, ${ }^{1,2}$ rheology control, ${ }^{3}$ and optoelectronic devices. ${ }^{4-6}$ Organosilanes are ideal candidates for such efforts due to their ability to self-assemble and the intrinsic hybrid configuration of organic/inorganic moieties. Yet, the main emphasis has been focused on alkylsilane, where an alkyl group is linked with a silane terminal, i.e., $\mathrm{R}-\mathrm{SiX}_{3}$ ( $\mathrm{R}$ is alkane and $\mathrm{X}$ can be halogen or alkoxy). Construction of stacked monolayers of alkylsilane through chemisorption is possible. The demonstrated pathway usually takes place after the monolayer surface is converted to a hydroxylated one. This conversion can be realized by a chemical modification of a nonpolar terminal group to a hydroxyl group. Alternatively, $\mathrm{Huo}^{7}$ and $\mathrm{Parikh}^{8}$ found that long-chain alkylsilane could form a lamellar solid or stacked bilayer through solvophobic and van der Waals interactions. Kuroda ${ }^{9,10}$ reported the chain-length dependence study $\left(\mathrm{C}_{n} \mathrm{H}_{2 n+1} \mathrm{Si}(\mathrm{OEt})_{3}, n=\right.$ $12,14,16$, and 18) of the bilayered structure. $\mathrm{Maoz}^{11}$ further developed a protocol to grow stacks of bilayers by manipulating the interactions in a stepwise fashion. Collectively, all these studies concluded that the solvophobic and the van der Waals interactions between molecules with long chains were the main driving forces for such a complex assembly. Due to the rather weak interactions between alkylsilanes, the resulting assemblies are usually amorphous and instable at high temperatures; the issue of disturbed packing, as well as difficulties in chemical functionalization of alkyl chains, limits the potential applications for organosilane lamellae.

In this communication, we report the aromatic silane molecules, 2-(4-pyridylethyl)triethoxysilane (PTS, a) and $\mathrm{N}$-(3-trimethoxysilylpropyl)pyrrole (b) (Chart 1), with dipoles on the aromatic ring could form a lamellae-like, stacked layer structure with the assistance of water, while the dipole-free phenethyltrichlorosilane (c) can not. Assembled structures by a and $\mathbf{b}$ kept layered stacking even at a high temperature of $350{ }^{\circ} \mathrm{C}$, of which we focus only on PTS in this communication. The SEM image (Figure 1 inset) clearly reveals a striated surface morphology suggestive of the presence of lamellae and their graphite-like features. The lateral sizes of the lamellae are in micrometers. Figure 1 shows the X-ray diffraction patterns of the solids of PTS. Two sharp peaks, centering at $\sim 20^{\circ}$ and $\sim 6.70^{\circ}$, correspond to $d$ spacings of $4.42 \AA$ and $14.28 \AA$, respectively. A $d$ spacing of $4.42 \AA$ can be roughly correlated to the lateral intermolecular spacing, which is different from the observed spacing in densely packed alkylsilanes. ${ }^{8,12}$ A $d$ spacing of $14.28 \AA$ is more intriguing and indicative of a lamellar motif. Calculated ordering (based on Scherer equation) in both the stacking and lateral directions are 44.0 and $9.0 \AA$, respectively. The presence

${ }^{\dagger}$ Department of Engineering Mechanics and Nebraska Center for Materials and Nanoscience, University of Nebraska.

* Department of Chemistry, University of Nebraska.

\& Army Research Laboratory.

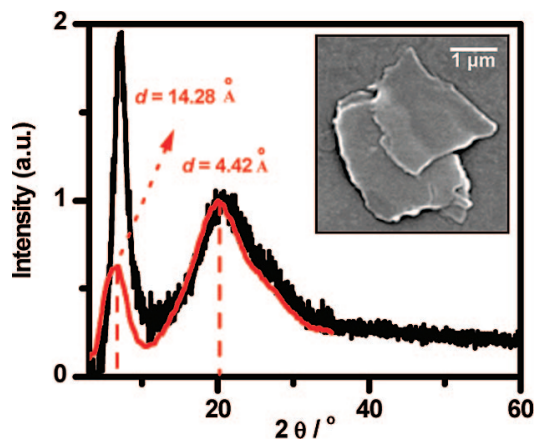

Figure 1. XRD and SEM (inset) of the PTS lamellae. Red line for the layer as formed; black line for the layer heated at $350{ }^{\circ} \mathrm{C} / 30 \mathrm{~min}$.

Chart 1. Chemical Structures of 2-(4-Pyridylethyl)triethoxysilane (a), $\mathrm{N}$-(3-Trimethoxysilylpropyl)pyrrole (b), and Phenethyltrichlorosilane (c) ${ }^{a}$
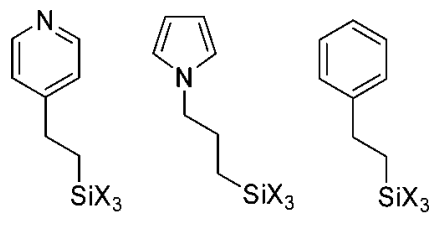

${ }^{a} \mathrm{X}$ can be an alkoxy or halogen.

of two peaks indicates one stacking pattern only. In contrast, alkylsilanes can adopt three possible stacking modes: mixed transgauche bilayer, all-trans bilayer, and all-trans interdigitated monolayer. ${ }^{13}$ The dipole-dipole interaction, as well as the rather short alkyl chain in PTS, could all have helped to leave out the up-anddown interdigitated pattern and to reduce the possibility of adopting a gauche conformation. On the other hand, replacing the heterocyclic ring with benzene (Chart 1 , c) canceled the dipole and changed the final product from a layered solid to a viscous gel. Lack of polarity in the latter compound certainly varied the interactions between molecules, which disfavored the lamellae formation process. These findings triggered our hypothesis that the dipole moment in an aromatic silane is a key player for the formation of lamellar stacking. Given the known richness of chemistry to functionalize aromatic rings, tailoring the chemical structure of the final lamellas can lead to many functional materials. This reported work may offer new opportunities for self-assembly and material design.

If the dipole in our polar silane (Chart $1, \mathbf{a}$ and $\mathbf{b}$ ) is assisting the self-assembly, it could play two active roles: (1) repulsion between the molecules and (2) attraction between the neighboring layers. In particular, when the water-induced silanolization or chemisorption takes place, individual silane molecules prefer a 


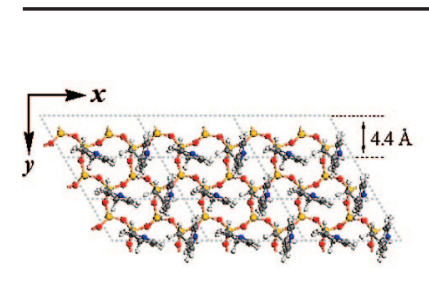

(a)

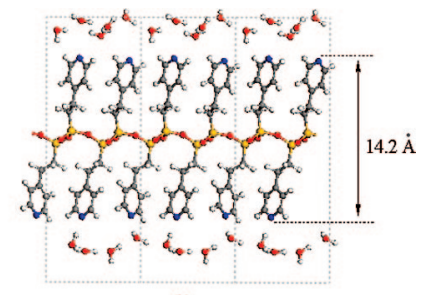

(b)

Figure 2. Optimized models of the PTS lamellae from the stacking of bilayers. Water molecules act as the interlayer binding agent.

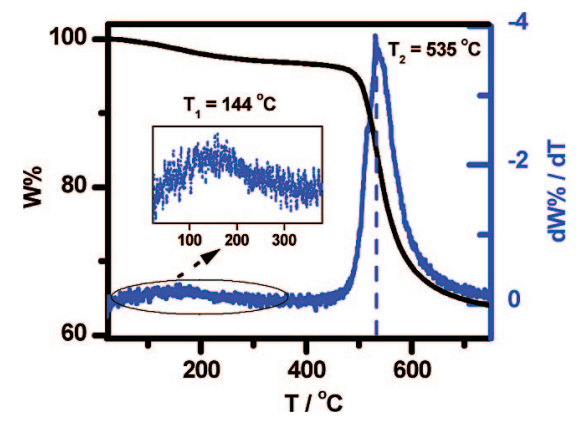

Figure 3. TGA of the PTS lamellae. Black line for the weight percent loss, and the blue line for derivative.

standing up orientation, i.e., parallel to the surface normal. Dipoles in those aromatic silanes, thus, become repulsive to each other. As a result, the hydrocarbon chain, as well as the aromatic ring, could have much less entanglement, leading to an ordered packing inside a monolayer. When such a monolayer is formed, extra water molecules in the organic solvent could be attracted by the permanent dipole in the layer. Therefore, a repetition of the above process generates stacked multilayers, as in the modeled packing shown in Figure 2 (computational details are given in the Supporting Information). We propose a lamellar or bilayer-by-bilayer stacking, with water sandwiched between two PTS bilayers. The polar groups are arranged in an alternating up-and-down motif (Figure 2b) to minimize the steric hindrances between adjacent pyridylethyl groups. The optimized thickness for the PTS bilayer is $14.2 \AA$ (in $z$-axis direction), which is in great agreement with the $d$ spacing 14.28 $\AA$ derived from XRD. Another $d$ spacing of $4.42 \AA$ derived from XRD is considered to correlate with the smallest periodic length in the layer plane ( $x y$ plane in Figure 2), measured at $4.4 \AA$ from the theoretical simulations. Simply this very length is the lateral intermolecular spacing aforementioned.

The large dipole on the polar aromatic ring provides strong electrostatic interactions between bilayers. The estimated binding energy between polar rings and water is $2.43 \mathrm{eV}$ per unit cell; however, the binding energy for the phenyl silane with water is only $1.74 \mathrm{eV}$ per unit cell. A repetition of these unit cells certainly could generate a much stronger binding between bilayers of pyridine compared to the phenyl ones. Such a strong interaction further resulted in thermally stable lamellar structures, which is confirmed by our combined thermogravimetric analysis (TGA) and XRD. The TGA profile (Figure 3) shows the presence of two discernible weight loss regions: a small transition at $144{ }^{\circ} \mathrm{C}$ and a large transition initiating around $450{ }^{\circ} \mathrm{C}$. Apparently, the weight loss above $450{ }^{\circ} \mathrm{C}$ is due to the decomposition of the organic moiety. The first transition at $144{ }^{\circ} \mathrm{C}$ (inset) is assigned as the loss of physically absorbed water. Whereas no additional weight loss was observed below $450{ }^{\circ} \mathrm{C}$, this very first transition peak could manifest only a moderate thermal resistance of the layered structure. However, further XRD analyses on our samples after the first peak in TGA clearly suggested otherwise, indicating a better packing and good thermal stability for the lamellae. The heated samples revealed an unchanged XRD pattern up to $350{ }^{\circ} \mathrm{C}$. More interestingly, the peak at $\sim 6.70^{\circ}$ in XRD showed an increased sharpness after the annealing (Figure 1, black plot). Very likely, annealing at an elevated temperature has helped the lamellae to exclude some of the redundant, physically adsorbed waters between layered stacks, which in turn promoted a more periodic and compact stacking. In contrast, most layered alkylsilanes ${ }^{4,12}$ either were transformed to a liquid-like phase or became amorphous upon heating at a temperature much lower than $350{ }^{\circ} \mathrm{C}$. It has been known that increasing the molecular dipole moment can effectively enhance the thermal stability of materials. We believe that the large dipole moment on the polar aromatic ring can also lead to strong electrostatic interactions between adjacent layers of pyridines and water molecules. This explains why the observed lamellar structure is thermally stable, as well as the absence of a layered stacking for the phenyl network.

In summary, aggravated van der Waals, $\pi-\pi$, and solvophobic interactions can all lead to the formation of lamellae. The importance of a dipole to the stacking is largely unknown. We report that a strong dipole-dipole interaction can be another important driving force for the lamellar formation. Controlled self-assembly of two polar aromatic silanes, i.e., pyridine- or pyrrole-terminated silane, supported this argument. Graphite-like features are clearly visible from SEM. In addition, XRD patterns suggest a $d$ spacing of $14.28 \AA$ along the $z$-axis and $4.42 \AA$ in the $x y$ plane, which are all in good agreement with our theoretical modeling. We expect that a future design of a series of polar molecules that will allow them to form rarely seen, thermally stable lamellae will add significantly to the body of knowledge regarding self-assembly and material design.

Acknowledgment. The authors thank Prof. Patrick H. Dussault and his group for their generous help and acknowledge the financial support from the NSF (CMMI 0825905) and the Army Research Office (W911NF-08-1-0190).

Supporting Information Available: A description of the experimental procedures and computational details. This material is available free of charge via the Internet at http://pubs.acs.org.

\section{References}

(1) Lagaly, G. Angew. Chem. 1976, 88, 628-639.

(2) Ijdo, W. L.; Lee, T.; Pinnavaia, T. J. Adv. Mater. 1996, 8, 79-83.

(3) Giannelis, E. P. Adv. Mater. 1996, 8, 29-35.

(4) Bourlinos, A. B.; Chowdhury, S. R.; Jiang, D. D.; An, Y.-U.; Zhang, Q.; Archer, L. A.; Giannelis, E. P. Small 2005, 1, 80-82.

(5) Peng, H.; Zhu, Y.; Peterson, D. E.; Lu, Y. Adv. Mater. 2008, 20, 11991204 .

(6) Yang, L.; Peng, H.; Huang, K.; Mague, J. T.; Li, H.; Lu, Y. Adv. Mater. 2008, 18, 1526-1535.

(7) Huo, Q. S.; Margolese, D. I.; Stucky, G. D. Chem. Mater. 1996, 8, 11471160.

(8) Parikh, A. N.; Schivley, M. A.; Koo, E.; Seshadri, K.; Aurentz, D.; et al. J. Am. Chem. Soc. 1997, 119, 3135-3143.

(9) Shimojima, A.; Sugahara, Y.; Kuroda, K. Bull. Chem. Soc. Jpn. 1997, 70, 2847-2853.

(10) Shimojima, A.; Wu, C. W.; Kuroda, K. J. Mater. Chem. 2007, 17, 658663.

(11) Maoz, R.; Matlis, S.; DiMasi, E.; Ocko, B. M.; Sagiv, J. Nature 1996, 384, 150-153.

(12) Fujii, K.; Fujita, T.; Iyi, N.; Kodama, H.; Kitamura, K.; Yamagishi, A. J. Mater. Sci. Lett. 2003, 22, 1459-1461.

(13) Shimojima, A.; Kuroda, K. Chem. Rec. 2006, 6, 53-63.

JA808103H 


\title{
Supporting Information
}

\section{Dipole-Induced, Thermally Stable Lamellar Structure by Polar}

\author{
Aromatic Silane \\ Jinyue Jiang ${ }^{1}$, Ocelio V. Lima ${ }^{1}$, Yong Pei ${ }^{2}$, Xiao Cheng Zeng ${ }^{2^{\star}}$, Li Tan ${ }^{1^{*}}$, and Eric \\ Forsythe \\ Department of Engineering Mechanics and Nebraska Center for Materials and \\ Nanoscience, University of Nebraska, Lincoln, NE, 68588 \\ ${ }^{2}$ Department of Chemistry, University of Nebraska, Lincoln, NE, 68588 \\ Flexbile Display Center, Army Research Laboratory, 2800 Powder Mill Road, \\ Adelphi, MA, 20783-1197
}

xzeng@unl.edu; ltan4@unl.edu; eric.forsythe@us.army.mil

\section{Table of Contents}

Experimental Details

Computational details

Figure S1. IR of PTS hydrolysis product

Figure S2. Modeled XRD peaks along the stacking direction of lamellae 


\section{Experimental Details}

Chemicals. Tetrahydrofuran (THF) and toluene were distilled in the bath of $\mathrm{K} / \mathrm{Na}$ under nitrogen before use. 2-(4-Pyridylethyl)triethoxysilane, N-(3-trimethoxysilylpropyl)pyrrole, phenethyltrichlorosilane, and other reagents were used as received from commercial suppliers. Water was purified with an Ultrapure Water System.

Thermalgravimetric Analysis (TGA). Thermally induced weight changes were monitored through Perkin-Elmer TGA-7. The measurements were made over the range $20-800^{\circ} \mathrm{C}$ at a heating rate of $10^{\circ} \mathrm{C} / \mathrm{min}$, and data were acquired at an interval of $0.15^{\circ}$.

X-ray Diffraction (XRD). Wide angle diffraction patterns were obtained on a Rigaku multiflex diffractometer (with D/max-2000 controller and a horizontal goniometer) using $\mathrm{Cu} \mathrm{K \alpha}(1.544 \AA)$ as radiation source. The samples ( $\sim 5 \mathrm{mg})$ were grinded into fine powders with a mortar and pestle, and spread as a thin layer on a zero-background holder. Data were recorded over a $2 \theta$ range of $5-60^{\circ}$ at an angular resolution of $0.04^{\circ}$.

General procedure to assemble 2-(4-pyridylethyl)triethoxysilane, N-(3-trimethoxysilylpropyl)pyrrole, and phenethyltrichlorosilane. A solution of $\mathrm{HCl}(3 \mathrm{M}, 6 \mathrm{~mL})$ was added to a $25 \mathrm{~mL}$ round-bottom flask, followed by dropwise addition of the above silane $(1 \mathrm{~mL})$ in Toluene $(2 \mathrm{~mL})$ via a syringe. The reaction was stirred at room temperature for $24 \mathrm{~h}$. The aqueous layer was neutralized with a solution of $\mathrm{NaOH}(3 \mathrm{M})$ to render the formation of 
precipitates. Then, the precipitates were collected after filtration, washed with water thoroughly and dried under vacuum.

\section{Computational Details}

The density functional theory (DFT) calculations are performed using DMol3 packages. ${ }^{1}$ The structure optimizations are performed using the generalized gradient approximation (GGA) with the Perdew-Burke-Ernzerhof (PBE) functional. ${ }^{2}$ The $d$-polarization included basis set (DND) is used. All-electron calculations are applied for $\mathrm{H}, \mathrm{C}, \mathrm{O}, \mathrm{N}$, and Si elements.

\section{References}

1. Delley, B. J. Chem. Phys. 1990, 92, 508; J. Chem. Phys. 2003, 113, 7756. $\mathrm{DMol}^{3}$ is available from Accelrys.

2. Perdew, J. P.; Burke, K.; Ernzerhof, M. Phys. Rev. Lett. 1996, 77, 3865. 


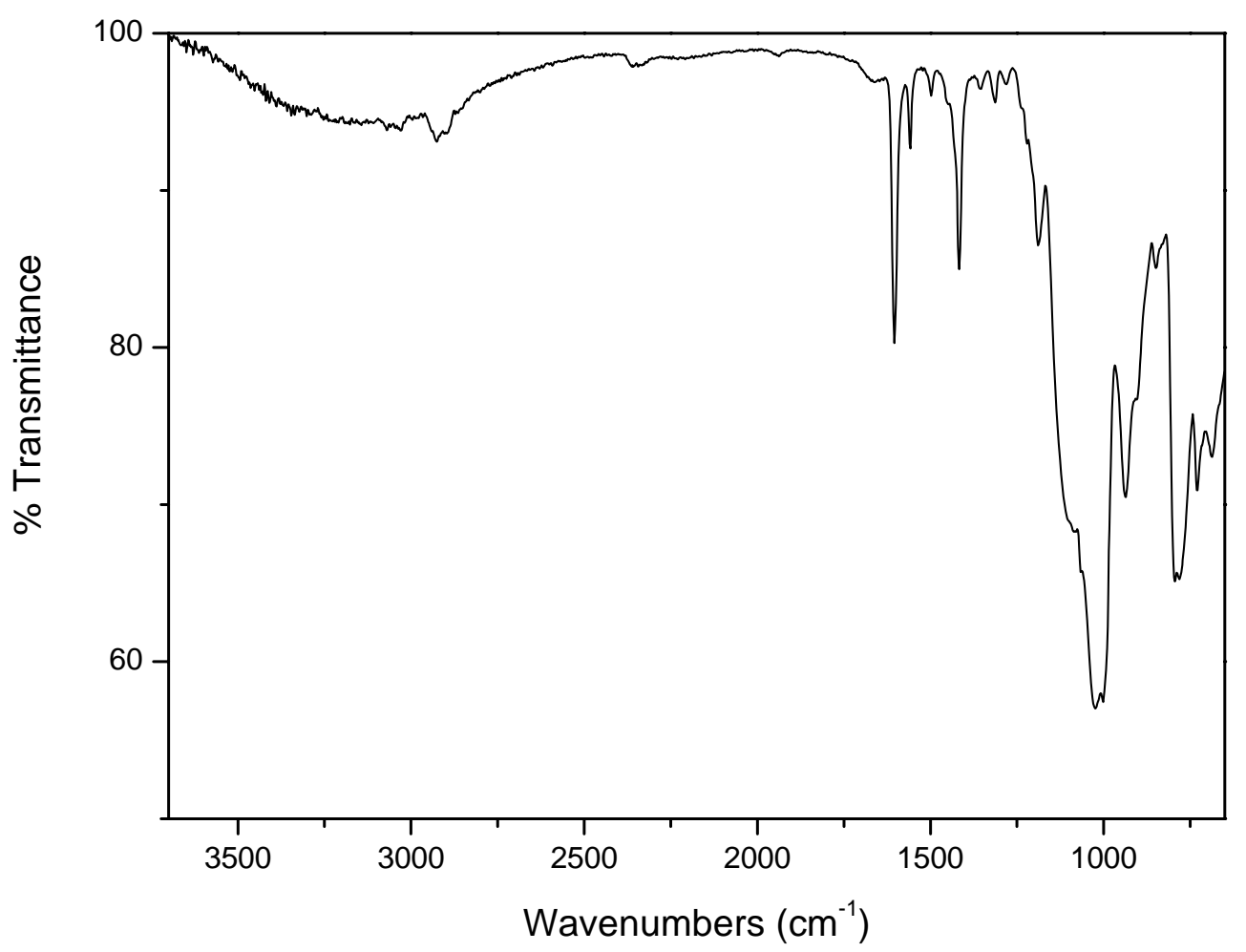

Figure S1. IR of PTS hydrolysis product. The strong peaks around 950 $-1150 \mathrm{~cm}^{-1}$ indicate polysiloxane networks, and the sharp peak around 1600 $\mathrm{cm}^{-1}$, pyridyl ring. 


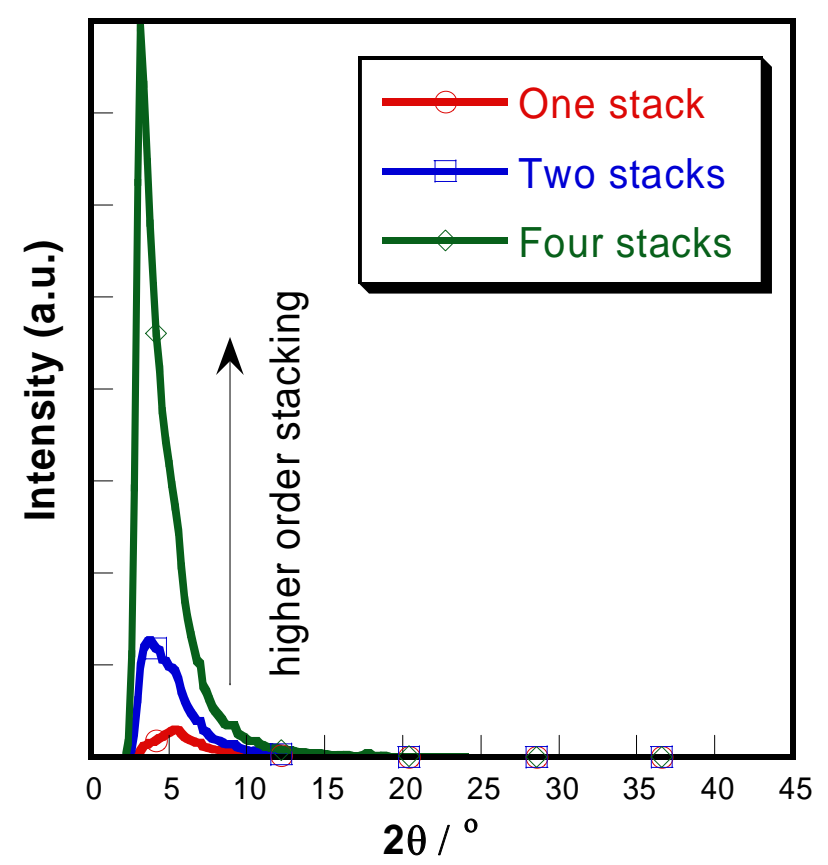

Figure S2. Modeled XRD peak along the stacking directions of the lamellae. This is to show that, at higher orders, no extra peaks at the small angles will show up. Rather, the peak intensity increases. 

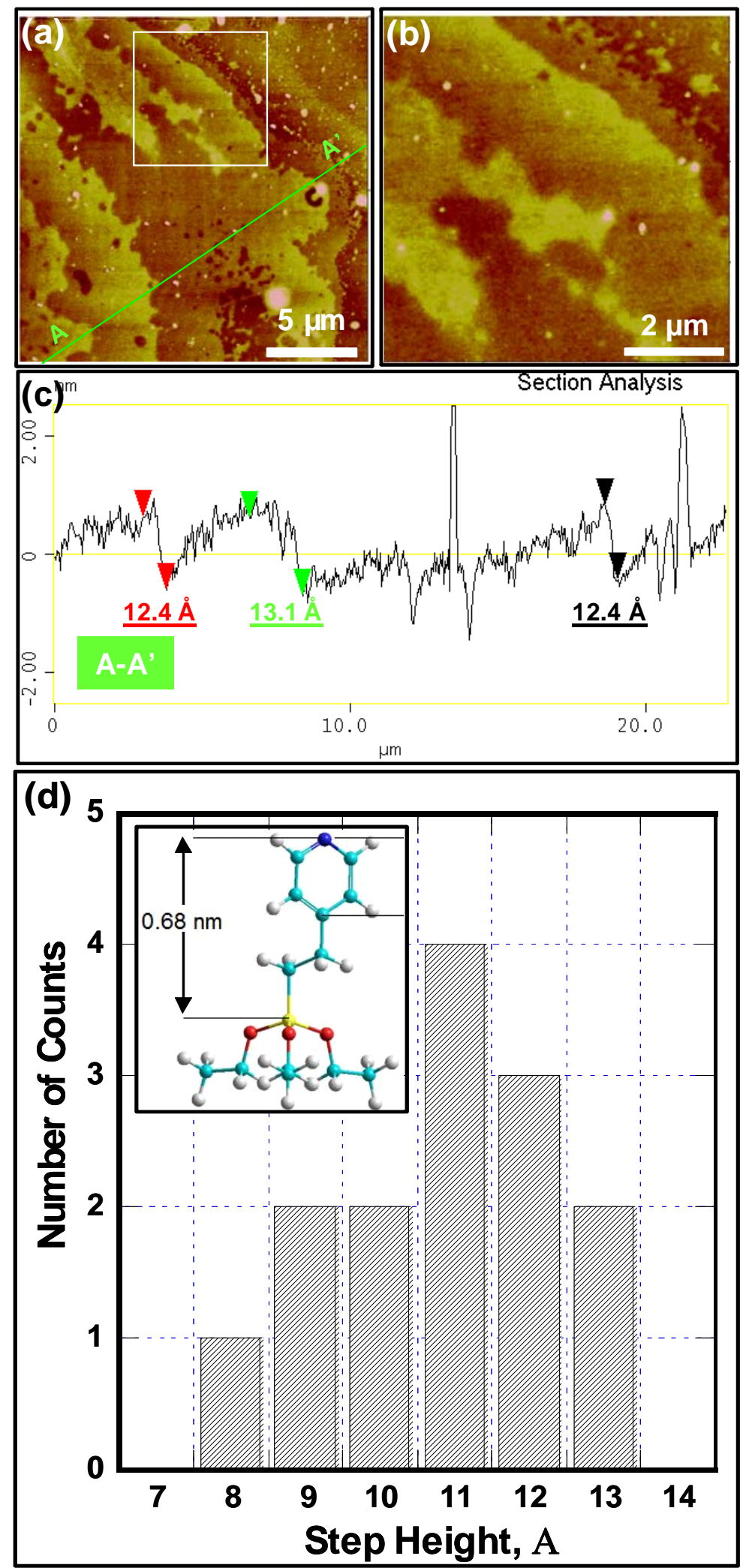

Figure S3. Topography of layered PTS and corresponding layer thickness. Atomic force microscopy images of the layered structure $(a \& b)$. To catch the initial stage of the layer formation, PTS molecules were allowed to 
self-assemble on surfaces of a silicon wafer. The wafer was intensively sonicated in isopropanol, acetone, and water for $15 \mathrm{~min}$ each. The assembly was processed by immersing the wafer in $0.3 \mathrm{mM}$ of PTS in toluene loaded with $5.0 \mu \mathrm{M} \mathrm{DI}$ water. Flat terraces of individual layers are clearly visible, indicating an organized stacking along the surface normal direction. Height profile in (c) showed a layer thickness around 12.0-13.0 nm. Histogram of 14 measurements across a sample surface of $20 \times 20 \mu \mathrm{m}^{2}$ showed the thickness of each layer varying between 8.0 and $13.0 \mathrm{~nm}$, centered at $11.0 \mathrm{~nm}$. The inset of (d) showed the estimated long dimension for the PTS molecule of 0.6-0.7 nm. Approximately, each layer in ( $\mathrm{a} \& \mathrm{~b}$ ) was composed by two vertically stacked molecules. Since the lamellae from the hydrolysis in Figure 1 were received from a solution, instead of surfaces, layers could have larger thickness than observed here. Overall, the data qualitatively match our bilayer-by-bilayer scheme showing in Figure 2. 


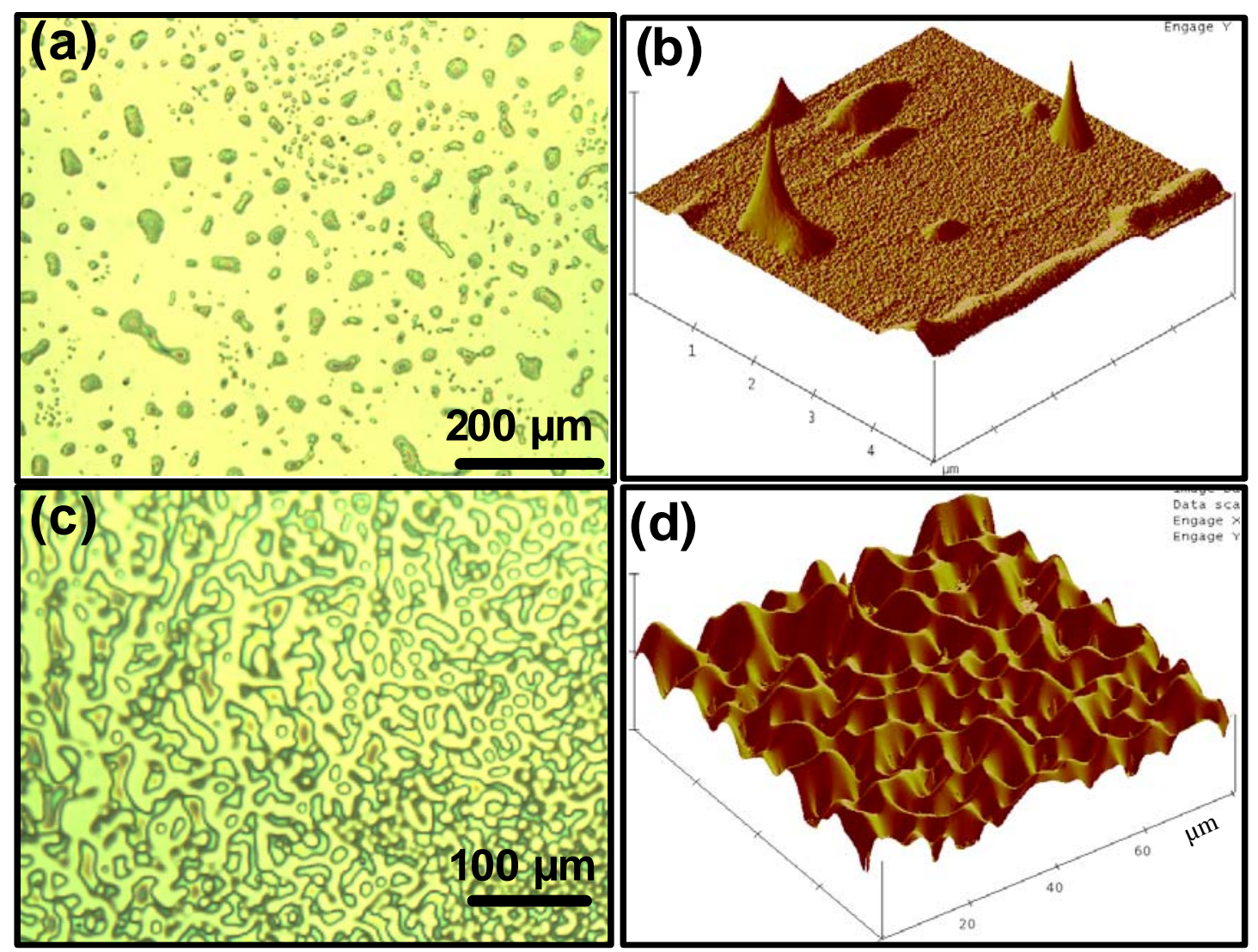

Figure S4. Morphology of the gels of phenethylsiloxane. Phenethyltrichlorosilane has no dipole on the aromatic ring. Hydrolysis product is a viscous gel, instead of a solid. The gel was dissolved in THF before drop casting over the surface of a silicon wafer. (a) The gels dewet on a hydrophobic wafer surface (optical microscopic image); (b) AFM image of the deweted, pointing-like features, showing no sign of layered stack; (c) the gels spread out rather evenly on a hydrophilic wafer surface (optical microscopic image); and (d) corresponding AFM image of the rough film, suggesting no sign of layers. 